\title{
Assessment of Stress-blended Eddy Simulation Model for Accurate Performance Prediction of Vertical Axis Wind Turbine
}

\author{
T.P. Syawitria,b), Y.F. Yao ${ }^{\text {a) }}$ J. $Y^{\left(o^{c}\right)}$ and B. Chandra ${ }^{\text {a) }}$ \\ ${ }^{a)}$ Department of Engineering Design and Mathematics, University of the West of England, Bristol BS16 1QY, \\ United Kingdom \\ ${ }^{b)}$ Department of Mechanical Engineering, Universitas Muhammadiyah Surakarta, Surakarta 57162, Central \\ Java, Indonesia \\ ${ }^{c}$ School of Engineering, University of Lincoln, Brayford Pool, Lincoln LN6 7TS, United Kingdom
}

Corresponding author:yufeng.yao@uwe.ac.uk

\begin{abstract}
Purpose

The aim of this paper is to assess the ability of a stress-blended eddy simulation (SBES) turbulence model to predict the performance of a three-straight-bladed vertical axis wind turbine (VAWT). The grid sensitivity study is carried out to evaluate the simulation accuracy.

Design/methodology/approach

The unsteady Reynolds-averaged Navier-Stokes (URANS) equations are solved by Computational Fluid Dyna mics (CFD) technique. Two types of grid topology around the blades, na mely O-grid (OG) and C-grid (CG) types are considered for grid sensitivity studies.
\end{abstract}

\section{Findings}

Simulation results have shown significant improvements of predictions by employing the SBES turbulence model than that of other turbulence models such as $k-\varepsilon$ model with regard to the power coefficient $\left(C_{p}\right)$. The $C_{p}$ distributions predicted by applying the CG mesh are in better agreement with the experimental data than that by the OG mesh.

Research limitations/implications

The current study provides some new insights of the use of SBES turbulence model in VAWT CFD simulation.

Practical implications

The SBES turbulence model can significantly improve the numerical accuracy on predicting the VAWT performance at lower tip speed ratio (TSR) for which other turbulence models cannot achieve. Furthermore, it has less computational demand for the finer grid resolution used in the RANS-Large Eddy Simulation (LES) "transition" zone, compared to other Hybrid RANS-LES models.

\section{Originality/value}

To authors' knowledge, this is the first attempt to apply SBES turbulence model to predict VAWT performance resulting in accurate CFD results. The better prediction can increase the credibility of computational evaluation of a new or an improving configuration of VAWT.

\section{Keywords}

Vertical axis wind turbine; stress-blended eddy simulation; grid topology sensitivity. 


\section{Nomenclatures}

A : area of the simulated model $\left(\mathrm{m}^{2}\right)$

$A_{s} \quad:$ rotor swept area $\left(\mathrm{m}^{2}\right)$

c : chord length $(\mathrm{mm})$

$C_{m} \quad:$ moment coefficient

$C_{p} \quad$ : power coefficient

$C_{\mu} \quad:$ : coefficient of turbulence viscosity formulation

Drotor : rotor diameter $(\mathrm{mm})$

$H_{\text {rotor }} \quad$ : rotor height $(\mathrm{mm})$

$H_{\text {wind }} \quad$ : wind tunnel height $(\mathrm{mm})$

$k \quad: \quad$ : turbulence kinetic energy $\left(\mathrm{m}^{2} / \mathrm{s}^{2}\right)$

$M \quad:$ moment force $(N)$

$R \quad: \quad$ turbine radius $(m)$, i.e. from the blade pressure centre to the supporting rod centre

$U_{\infty} \quad:$ : wind speed $(\mathrm{m} / \mathrm{s})$

$W_{\text {wind }} \quad$ : wind tunnel wide $(\mathrm{mm})$

Greek symbols

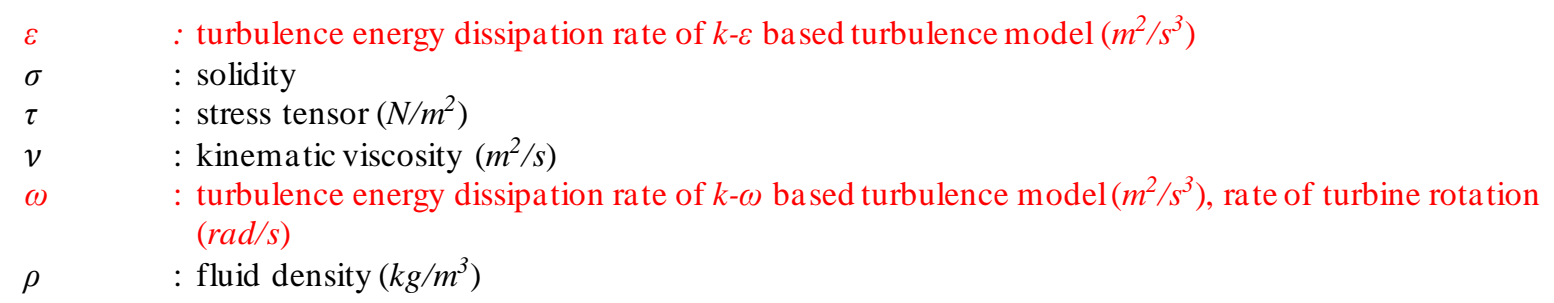

\section{Subscripts}

$i, j \quad:$ grid position in $x$ and $y$ coordinates respectively

$t \quad$ : turbulence 


\section{Introduction}

High-cost of power grid installation and connection have led to the growing interests in developing effective and affordable wind turbines in urban areas. While Horizontal Axis Wind Turbines (HAWTs) are still dominant in wind power industry, they gradually become less competitive in urban areas than those Vertical Axis Wind Turbines (VAWTs). Compared to HAWTs, VAWTs benefit from lower installation and maintenance costs (Ghasemian, et al., 2017). Furthermore, as VAWT can operate with any wind directions, they do not need a dedicated yaw mechanism. This can largely increase the operational reliability and thus is more suitable for urban environment where multidirectional wind flow exists (Sutherland, et al., 2012). In addition, their resilient characteristics to the wake effect of upstream blades and the vibrant background turbulence have made them preserving the ability to generate more power than HAWTs in urban areas (Dabiri, 2011).

While the performance of wind turbine can be measured by full-scale model for on-site tests or reducedscale model for wind tunnel experiments, there are growing trends to use ComputationalFluid Dynamics (CFD) method to predict the turbine performance such as the power generation. One major challenge in CFD simulation of VAWTs is to model turbulent flow around the rotating blades and the wake-turbulence interactions. The majority of turbulence models are able to capture the time-a veraged mean flow properties with steady Reynoldsaveraged Navier-Stokes (RANS) approach, while the large-scale flow unsteadiness can be reproduced using unsteady RANS (URANS) simulations. Both are sufficient for most engineering applications. However, the RANS model could not capture the small-scale turbulence fluctuations, which are important for understanding the underlying flow physics. This could affect the accuracy of CFD predictions for VAWT performance. Hence, more advanced and robust turbulence models are needed in evaluating the turbine blade performance.

Most CFD studies of VAWTs have utilized the two-equation turbulence models such as the $k$ - $\varepsilon$ model and its variants. In particular, the realizable $k-\varepsilon$ model (which compared to standard $k-\varepsilon$ model, the constants in turbulence viscosity formulation, $C_{\mu}$, is not constant but a variable and the dissipation rate, $\varepsilon$, is derived from an exact equation for the transport of the mean-square vorticity fluctuation) is commonly used as it can produce reasonable good results for swirling flows, rotating and separating flows, boundary layers under strong adverse pressure gradients, and separated and recirculated flows, compared to the standard $k$ - $\varepsilon$ model (Castelli, et al., 2010; Castelli, et al., 2011; Trivellato \& Castelli, 2014; Mohamed, et al., 2015). For example, the results by (Castelli, et al., 2011) demonstrated that the realizable $k-\varepsilon$ model can predict the power coefficient $\left(C_{p}\right)$ and the optimum ratio between the tangential speed of the tip of a blade and the actual speed of the incoming wind, Tip Speed Ratio (TSR), in good agreements with the experimentaldata, even though it overestimated the power coefficient in the lower TSR range by a factor of 2 compared to test data. The simulation of (Ferreira, et al., 2010) also showed that the standard $k-\varepsilon$ turbulence model was able to predict the time-averaged vertical velocity distributions and the roll-up of the trailing-edge vortex shedding at the right phase angle (at around $120^{\circ}$ azimuthal position), compared to that produced by the Spalart-Allmaras $(S A)$ turbulence model.

Another two-equation turbulence model often utilized in VAWTs simulation is the $k$ - $\omega$ shearstress transport ( $k-\omega$ SST) model. For example, the study by (Wang, et al., 2018) showed that the $k-\omega$ SST model could produce the $C_{p}$ curve in alignment with the experimental results, by improving the prediction errors about $50 \%$ at low TSR and about $35 \%$ at high $T S R$ ranges respectively, compared to the realizable $k-\varepsilon$ model. Similar results also obtained by other researchers using the $k$ - $\omega$ SST turbulence model (Lam \& Peng, 2016; Almohammadi, et al., 2015; Arab, et al., 2017). In addition, a few studies have applied Transition SST turbulence model (Lanzafame, et al., 2014; Bangga, et al., 2017a; Rezaeiha, et al., 2018). Compared to two-equation models, the prediction accuracy of Transition SST turbulence model was generally improved. While the predicted power coefficients were close to the experimentaldata in the low TSR range, it still overestimated the $C_{p}$ value in the high $T S R$ range. The cause of this discrepancy has not been fully understood yet.

To further improve the prediction accuracy, more advanced turbulence models, such as Large Eddy Simulation (LES), have to be used in VAWTs simulation. The LES model is based on spa tially filtered equations thus it is accurate in time. It explicitly calculates the large-scale eddies which contain the majority of energy spectrum, which affects the main flow. For small-scale eddies, their effects on the flow are considered using a Sub-grid Scale (SGS) model due to the universal behaviour of turbulence (i.e. Kolmogorov hypothesis). This feature makes the LES model more suitable to predict the behaviour of the vortices a ssociated with the large flow separation and the dynamic stall in VAWTs. Despite of its capability for better prediction of flow characteristics around rotating bodies such as VAWTs, LES study for VAWTs applications are still very limited, mainly due to 
its expensive computational cost (Li, et al., 2013; Ghasemian \& Nejat, 2015; Elkhoury, et a1., 2015; Posa \& Balaras, 2018). To overcome this difficulty, a hybrid RANS-LES model like Detached Eddy Simulation (DES), Improved Delayed Detached Eddy Simulation (IDDES), and Wall-Modelled LES (WM-LES) are developed and utilized by many researchers in VAWTs applications (Lam \& Peng, 2016; Lei, et al., 2017; Peng \& Lam, 2016). These models still utilize RANS turbulence model in the nearwall region to model small eddies, while switching to LES to accurately simulate large eddies in the intermediate and the far flow fields, including separated shear layer and wake regions (Liu, et al., 2017).

Recently, a Stress-blended Eddy Simulation (SBES) turbulence model has been developed by (Menter, 2018). This is an improved turbulence model based on DES and/or IDDES concept, to address some numerical issues observed in previous DES such as Grid-induced Separation (GIS). The latter is mainly due to the tendency of 'shielding' the boundary layer to be solved with the RANS mode and slowly "transition" from the RANS zone to the LES zone in Separating Shear Layers (SSLs) (Frank \& Menter, 2017). Instead, the SBES model uses an improved 'shielding' function to protect the RANS boundary layers and to switch to an existing algebraic LES model in the LES zone. As a result, the RANS and the LES zones can be clearly distinguished by visualizing the 'shielding' function. Moreover, due to the lower turbulence stress level enforced by the LES model, the SBES model can reduce the transition time from the RANS to the LES in SSLs, which can produce better, realistic, and consistent solutions. Furthermore, this turbulence model allows a RANS-LES "transition" even on a coarser grid that other DES models cannot.

To authors' knowledge, it is not yet found in public domain about the application of SBES turbulence model for CFD simulation of VAWTs. Nevertheless, this turbulence model has been successfully applied in the CFD simulations of rotating devices (Ravelli \& Barigozzi, 2018a; Ravelli \& Barigozzi, 2018b; Cai, et al., 2019). They reported that SBES model can produce the better predictions compared to other hybrid RANS-LES models (Ravelli \& Barigozzi, 2018b). Furthermore, SBES model can generate finer turbulence structures with abundant vortex structures. In comparison with other hybrid RANS-LES models, SBES model can capture faster development of turbulence and more ordered turbulence structures (Cai, et al., 2019). Hence, this paper will be the first attempt of this kind to simulate a three-straight-bladed VAWT using the SBES turbulence model in order to access its capability of producing accurate CFD prediction and to evaluate its sensitivity on grid topology change.

\section{Methodology}

2.1 The Vertical Axis Wind Turbine (VAWT) model

Two-dimensional (2D) simulations of a three-straight-bladed VAWT featured in a study by (Castelli, et al., 2011) (see Figure 1) are carried out. 2D simulation is chosen to decrease the computational cost as this study only limited to evaluate the ability of SBES turbulence model to predict flow around VAWT. Even though threedimensional (3D) simulation is preferable for flow with separation, previous study using hybrid LES-RANS model(DES) showed that 2D simulation could generate similar results with 3D simulation in turbulent separation case (Travin, et al., 2000). Hence, it is reasonable to perform 2D simulation by using SBES turbulence model as the model is actually based on DES model.

The blades use NACA 0021 a erofoil as cross-section profile. Three turbine blades rotate in counterclockwise direction around a supporting rod in the centre. The pressure centre of the blade is set at 0.25 chord from the leading edge of the aerofoil (The place where the rod is connected to the blade). The geometry details are summarized in Table 1. The rotor blade azimuthalposition is calculated based on the angular coordinate from the pressure centre of blade 1. In the experiments performed by (Castelli, et al., 2011), the turbine was operated at several angular velocities $(\omega)$ with a constant incoming wind speed $\left(U_{\infty}\right)$ of $9 \mathrm{~m} / \mathrm{s}$. The angular velocity $\omega$ is used to define $T S R$ as,

$T S R=\frac{\omega R}{U_{\infty}}$,

where $R$ is the turbine radius, i.e. from the blade pressure centre to the supporting rod centre. 


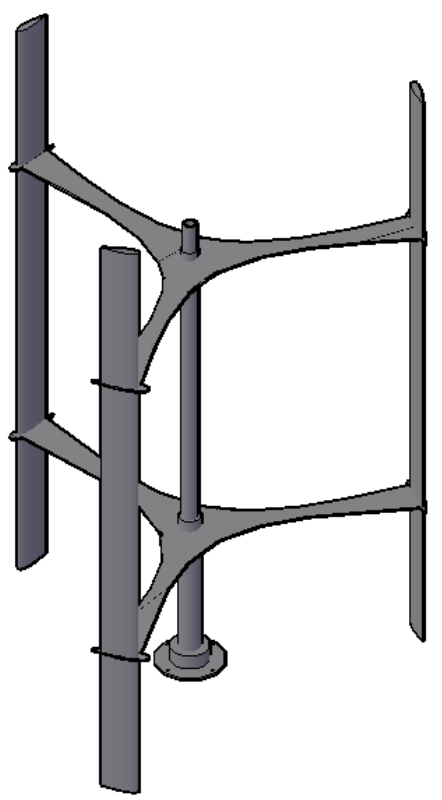

Figure 1. ExperimentalVAWT model (all measurements can be seen in Table 1) based on (Castelli, et al., 2011).

\subsection{Turbulence modelling}

This CFD investigation uses SBES turbulence model, which revises the shielding function of the shielded DES (SDES) SST model to switch between the LES and the RANS zones automatically. While the blending function remains the same as that of the shielding function SDES $\left(f_{S D E S}\right)$, in the LES zone where $f_{S D E S}=0, \operatorname{SBES}$ introduces an explicit model switching to an algebraic LES. By adding equation (2) shown below, the SBES model achieves a smooth blending of the Reynolds stress between the RANS and the LES formulations (Frank \& Menter, 2017) as:

$\tau_{i j}^{S B E S}=f_{S D E S} \tau_{i j}^{R A N S}+\left(1-f_{S D E S}\right) \tau_{i j}^{L E S}$

where $\tau_{i j}^{R A N S}$ is the RANS Reynolds stress tensor and $\tau_{i j}^{L E S}$ is the LES stress tensor. If the model is based on the eddy viscosity concept, this equation can be further simplified as:

$v_{t}^{S B E S}=f_{S D E S} v_{t}^{R A N S}+\left(1-f_{S D E S}\right) v_{t}^{L E S}$

For the RANS model, the transition SST turbulence model will be used.

Table 1. Main geometrical features of experiment model (Castelli, et al., 2011).

\begin{tabular}{|c|c|}
\hline Rotor diameter $\left(D_{\text {rotor }}(\mathrm{mm})\right)$ & 1030 \\
\hline Rotor height $\left(H_{\text {rotor }}(\mathrm{mm})\right)$ & 1456.4 \\
\hline Rotor swept area $\left(A_{s}\left(\mathrm{~m}^{2}\right)\right)$ & 1.236 \\
\hline Number of blade $(N(-))$ & 3 \\
\hline Blade profile & NACA 0021 \\
\hline Chord length $(c(\mathrm{~mm}))$ & 85.8 \\
\hline Spoke-blade connection & $0.5 \mathrm{c}$ \\
\hline Solidity $(\sigma(-))$ & 0.5 \\
\hline Wind tunnel height $\left(H_{\text {wind }}(\mathrm{mm})\right)$ & 4000 \\
\hline Wind tunnel wide $\left(W_{\text {wind }}(\mathrm{mm})\right)$ & 8000 \\
\hline
\end{tabular}


Table 2. Simulation setup

\begin{tabular}{|l|l|}
\hline \multicolumn{1}{|c|}{ Parameters } & \multicolumn{1}{|c|}{ Current Simulation } \\
\hline Fluid & Incompressible \\
\hline Model & 2D \\
\hline Solver & Pressure-based; Unsteady \\
\hline Models & Transitional SST SBES \\
\hline \multirow{5}{*}{ Methods } & Pressure-Velocity Coupling \\
\cline { 2 - 2 } & Scheme : SIMPLE \\
\cline { 2 - 2 } & Spatial Discretization \\
\cline { 2 - 2 } & Gradient : Least Squares Cell Based \\
\cline { 2 - 3 } & Pressure : Standard \\
\cline { 2 - 2 } & Momentum : Bounded Central Differencing \\
\cline { 2 - 3 } & Turbulence Kinetic Energy: Second Order Upwind \\
\cline { 2 - 3 } & Specific Dissipation Rate: Second Order Upwind \\
\cline { 2 - 3 } & Intermittency: Second Order Upwind \\
\cline { 2 - 3 } & Momentum Thickness Re: Second Order Upwind \\
\cline { 2 - 3 } & Transient Formulation : Second Order Upwind \\
\hline Residual convergence criterion & $10^{-6}$ \\
\hline Initialization & Hybrid Initialization \\
\hline Iteration every time step & 40 \\
\hline Time Step & $1^{\circ}$ \\
\hline Number of revolutions & 34 \\
\hline
\end{tabular}

\subsection{Simulation setup}

The simulation runs in unsteady mode using a pressure-based solver. Table 2 provides the details of solution methods and settings. All residuals are set to be $10^{-6}$. According to the numerical study by (Castelli, et al., 2011), the rotor height and the rotor swept area are adjusted to be $1000 \mathrm{~mm}$ and $1.03 \mathrm{~m}^{2}$ respectively for 2D modelling, and the time step is set to equal $1^{\circ}$ rotation of the rotor blade. The totalsimulation time is determined by the rotor revolution, which should be sufficiently long in order to allow the wake unsteadiness and the periodic motion of the rotating blades to be fully developed.

Table 3. Grid discretization

\begin{tabular}{|c|c|c|}
\hline & O-grid & C-grid \\
\hline \multicolumn{3}{|l|}{ Type of Shape } \\
\hline Far field & Rectangular & C combined with rectangular \\
\hline Rotating Core & Circle & Circle \\
\hline Control Circle & Circle & C combined with rectangular \\
\hline \multicolumn{3}{|l|}{ Type of Grid } \\
\hline Far field & Quadrilateral structured grid & Quadrilateral structured grid \\
\hline Rotating Core & Quadrilateral dominant grid & Quadrilateral dominant grid \\
\hline Control Circle & Quadrilateral structured grid & Quadrilateral structured grid \\
\hline \multicolumn{3}{|l|}{ Total number of cells } \\
\hline Far field & 34200 & 18240 \\
\hline Rotating Core & 22527 & 22527 \\
\hline Control Circle & 20880 & 49680 \\
\hline Growth Rate & 1.2 & 1.2 \\
\hline Element around body & 174 & 174 \\
\hline Element around trailing edge & 14 & 14 \\
\hline Body sizing for rotating core & $12 \mathrm{~mm}$ & $12 \mathrm{~mm}$ \\
\hline
\end{tabular}




\subsection{Computational domain and grid discretization}

The study starts with grid sensitivity analysis with two types of grid (denoted as O-grid (OG) and C-grid (CG) thereafter). Table 3 lists the details of the grid discretization for these two grids. The aim is to identify an appropriate grid topology and resolution for the main VAWT simulation using SBES turbulence model. Figure s 2 and 3 show the computational domain which consists of three sub-domains, namely farfield, rotating core and control sub-domains for OG and CG meshes, respectively. The specifications of the domain and the grid generation are described below.

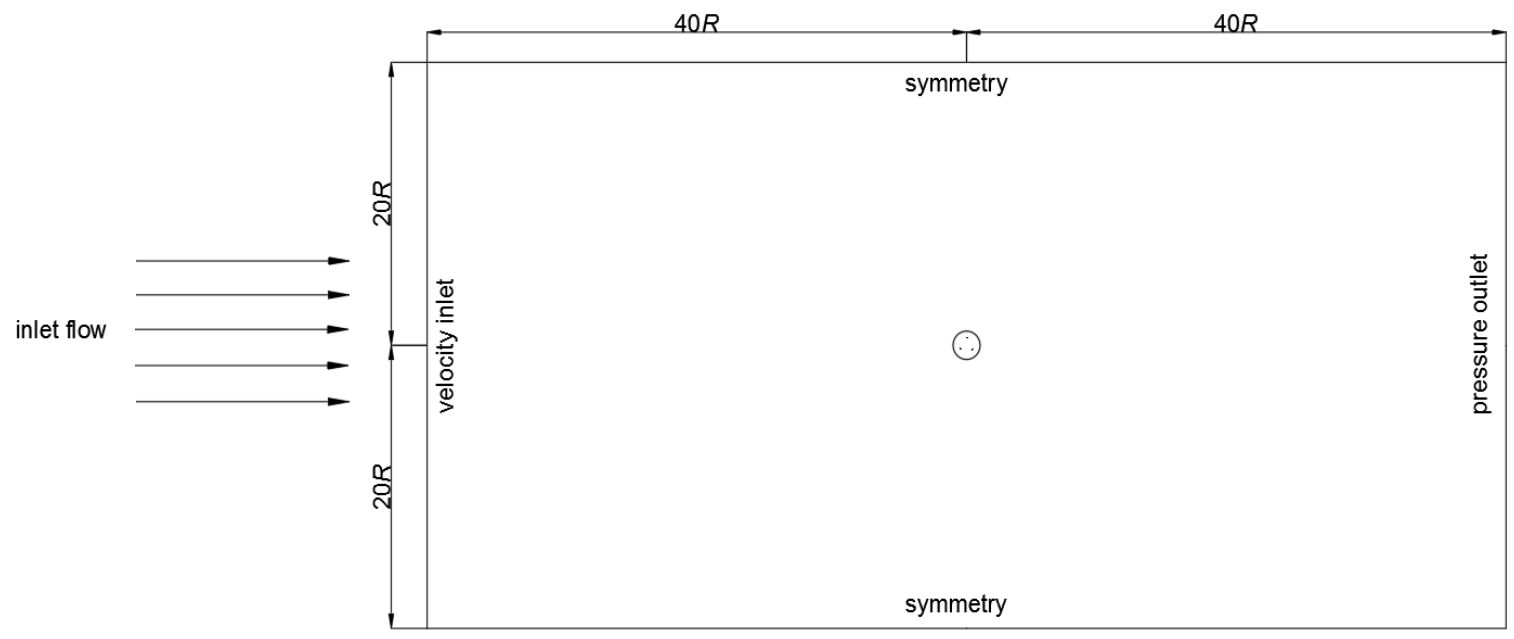

(a) Overview of the computationaldomain

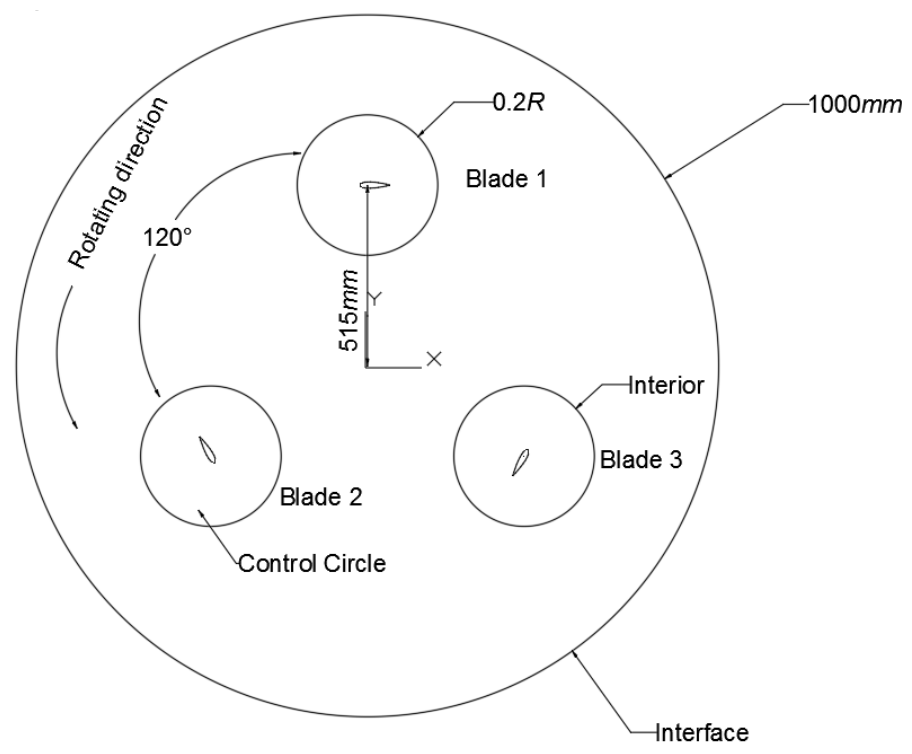

(b) Rotating core sub-domain

Figure 2. Detailed computational domain and sub-domains of O-grid. 


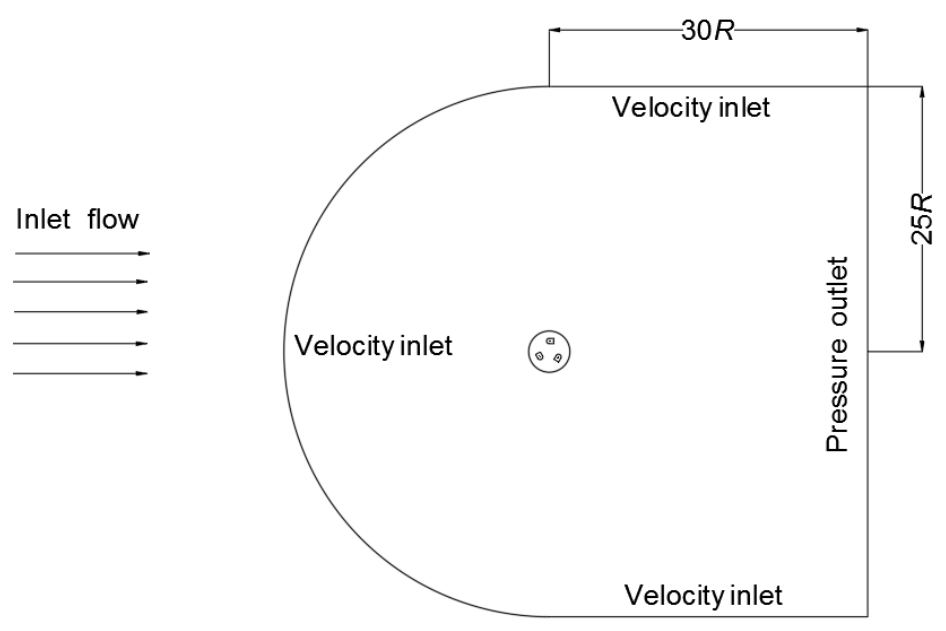

(a) Overview of the computationaldomain

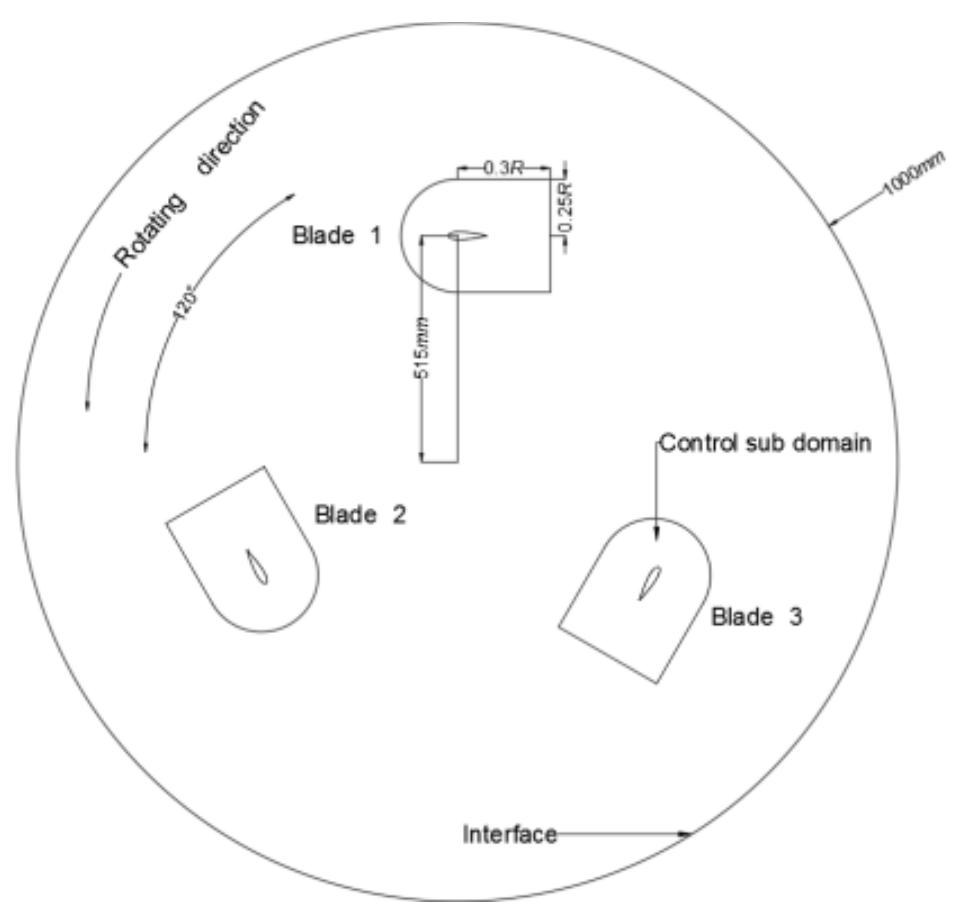

(b) Rotating core sub-domain

Figure 3. Detailed computational domain and sub-domains of C-grid.

\subsubsection{Far field sub-domain}

This is non-rotating sub-domain surrounding the rotating core sub-domain. It has different shapes for each type of grid as explained below.

O-grid

The O-grid uses rectangular far field sub-domain as suggested in previous studies (Castelli, et al., 2011; Wang, et al., 2018; Lam \& Peng, 2016). Based on (Wang, et al., 2018), to a void the boundary condition influences, the inlet and the outlet boundaries are located at $40 R$ a way from the centre of the domain, while the side walls are located at $20 R$ a way from the centre of turbine rotating axis, respectively. The velocity inlet and the pressure outlet boundary conditions are applied. Meanwhile, the side walls are defined as symmetric boundaries. A structured grid with quadrilateralcells is generated in this sub-domain (see Figure 4a). 
The $\mathrm{C}$ shape together with a rectangular enclosure is used for the farfield sub-domain. The $\mathrm{C}$ shape has $25 R$ in radius and the rectangular enclosure has $30 R$ in stream wise distance from the centre of the turbine rotating axis to the exit, as suggested by (Zhu, et al., 2018). Figure 5a has shown this sub-domain is divided into 6 regions to facilitate smooth grid discretization. Similar to the O-grid, a structured grid with quadrilateralcells is generated within this sub-domain (see Figure 5b).

\subsubsection{Rotating core sub-domain}

This sub-domain is fluid region and utilised to implement the revolution of the rotor blade. It has $2000 \mathrm{~mm}$ in diameter and rotates in anti-clockwise direction a round the turbine rotating ax is at a given angular velocity. To ensure the continuity of fluid flow in the farfield and the rotating core sub-domains, a 'fluid-fluid' interface is set up at the boundary intersection of these two sub-domains. The two types of grid both utilise quadrilateraldominant elements (see Figures $4 b$ and $5 c$ ).

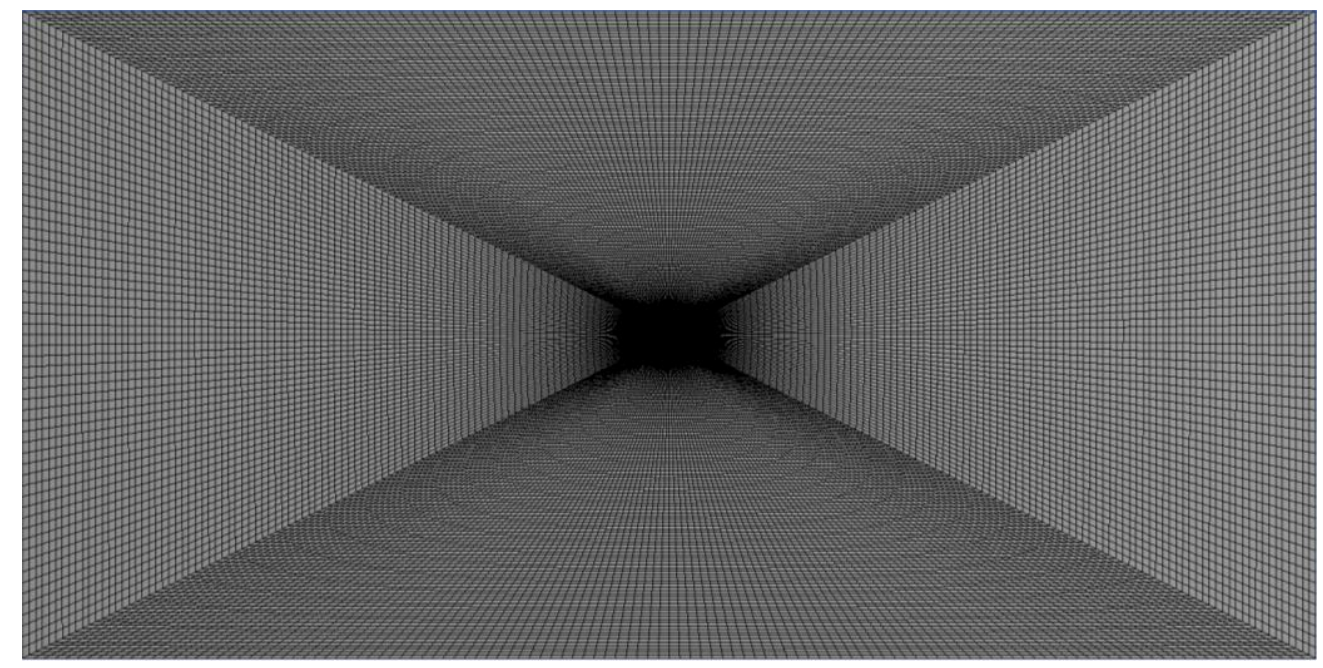

(a) Far field

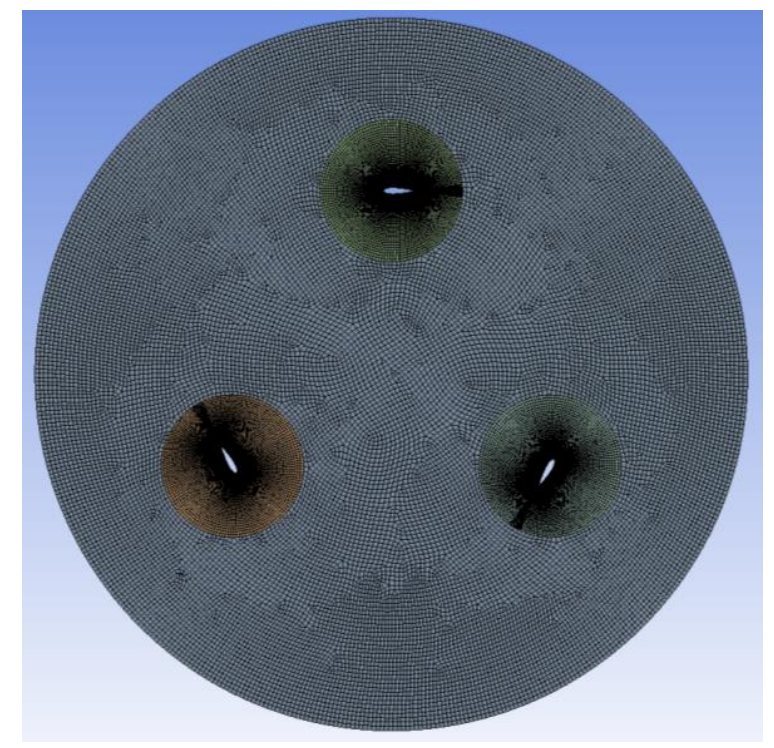

(b) Rotating core

Figure 4. Grid details in two sub-domains of O-grid. 


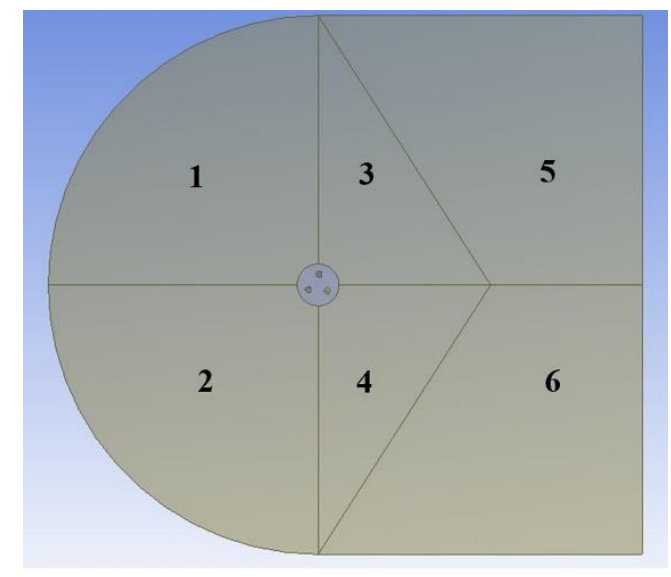

(a) Partition of farfield sub-domain

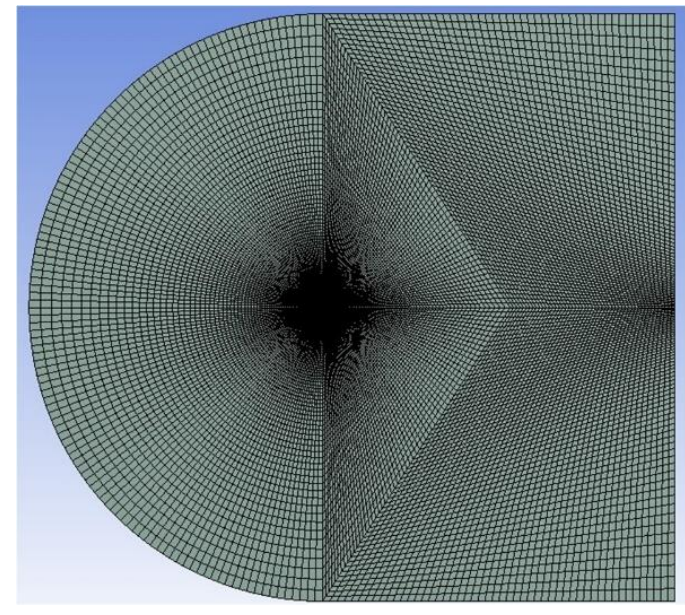

(b) Far field sub-domain

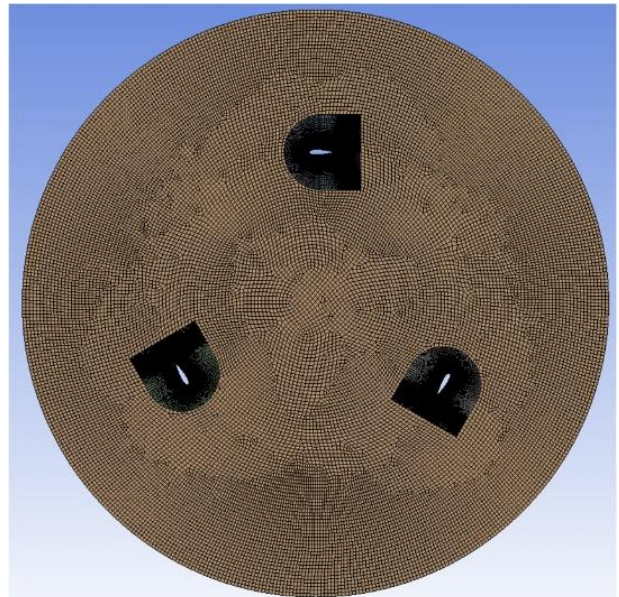

(c) Control sub-domain

Figure 5. Grid details in two sub-domains of C-grid.

\subsubsection{Control sub-domain}

This sub-domain is used to generate meshes around the blades. Three control domains with inserted blades are located inside the rotating core and separated by $120^{\circ}$ angular distance between the adjacent blades. The boundary is also interpreted as "interior" to ensure the continuity of the fluid flow.

For OG, each control sub-domain has a circle shape with a radius of $200 \mathrm{~mm}$, in which a structured O-grid around a blade is generated. For $\mathrm{CG}$, the $\mathrm{C}$-shape has $0.25 R$ in radius and $0.3 R$ in length from the centre of the blade. It uses the $\mathrm{C}$-grid around the blade with gradually increased grid size.

The structured quadrilateral cells are generated in this sub-domain, with fine grids in the near wall region (see Figures 6a and 6b) and coarse grids a way from the wall. When transition SST turbulence model is used, it is necessary to generate the first layer height to satisfy the criteria of non-dimensional wall distance $\Delta y_{1}{ }^{+}<1$. 


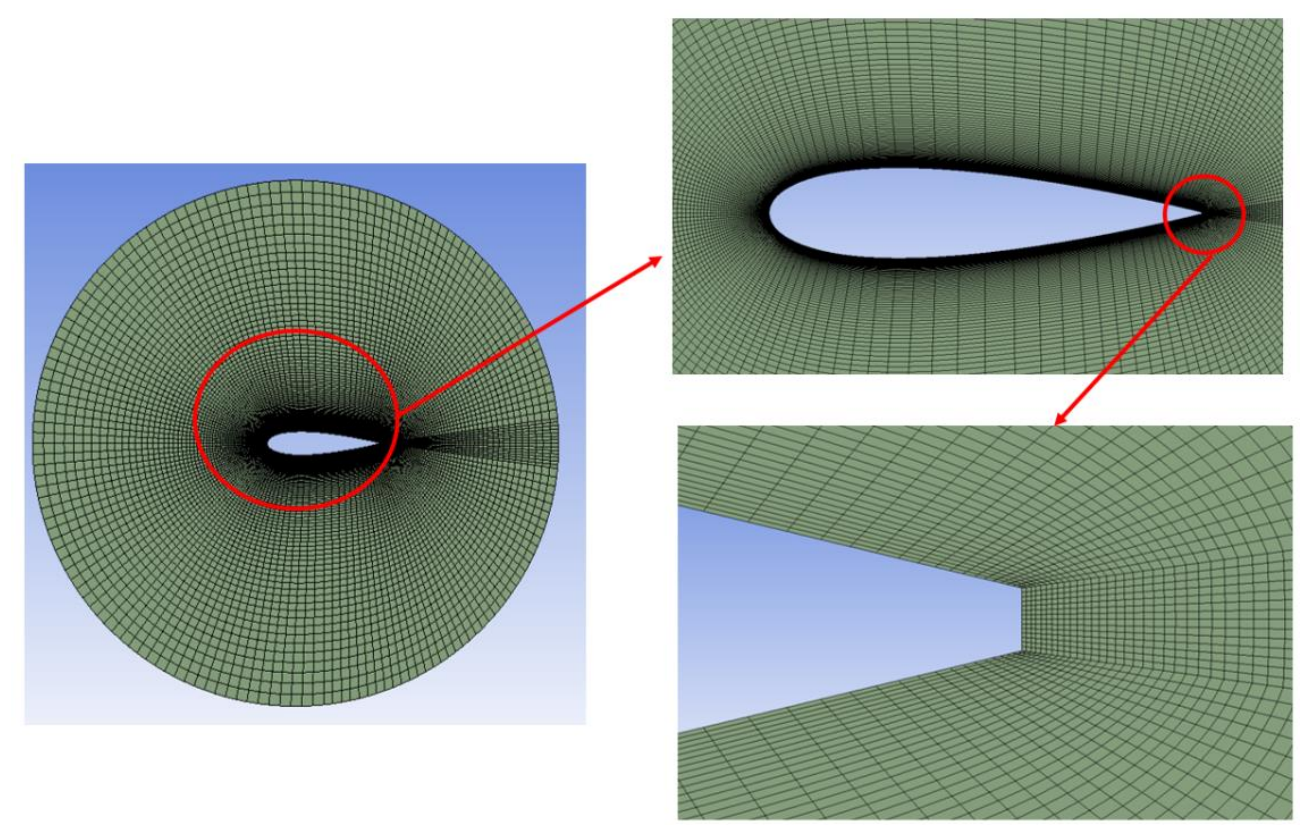

(a) O-grid

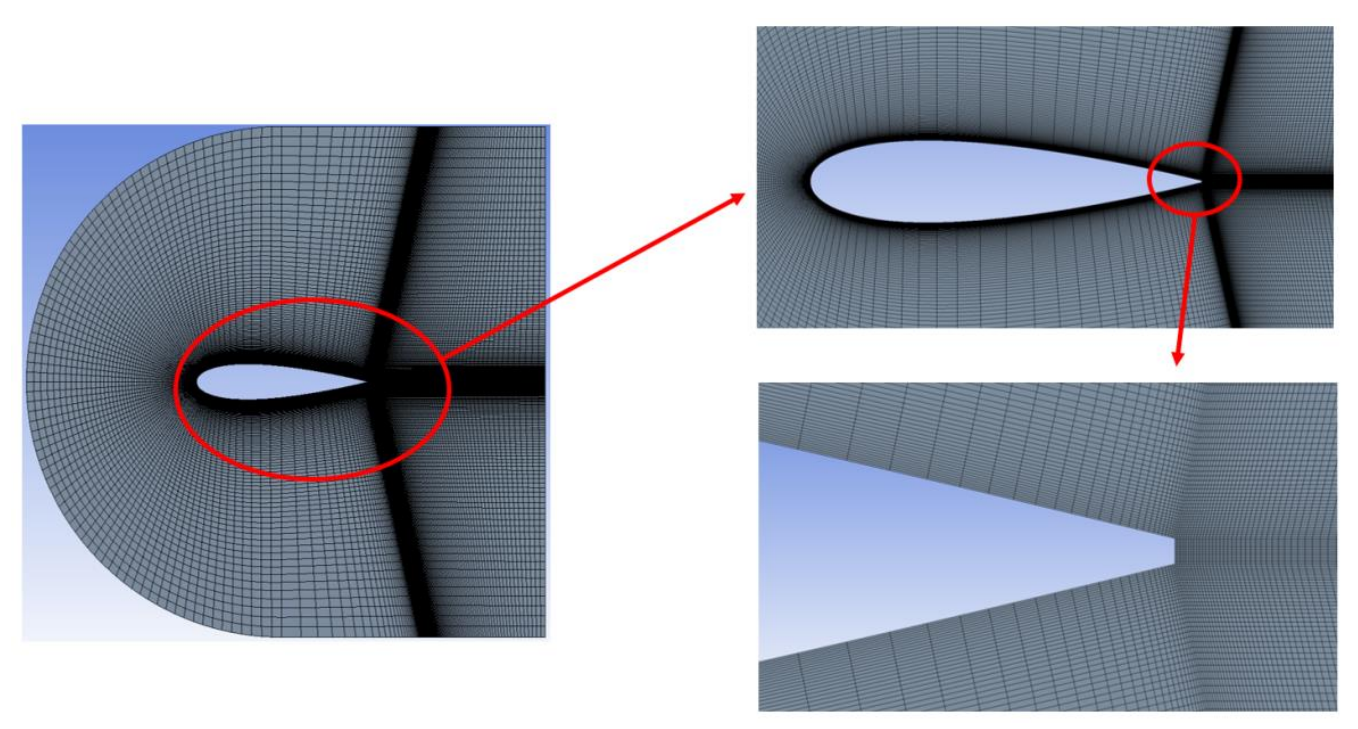

(b) C-grid

Figure 6. Grid around blade wall.

\section{Results and Discussion}

3.1 Revolution convergence

In VAWT simulation, it is necessary to collect data samples after reaching a statistically converged flow field. This can be done by monitoring time history of the moment coefficient $\left(C_{m}\right)$ or the power coefficient $\left(C_{p}\right)$ defined as:

$$
\begin{aligned}
& C_{m}=\frac{M}{\frac{1}{2} \rho U_{\infty}^{2} A_{s} R}, \\
& C_{p}=T S R \times C_{m},
\end{aligned}
$$


where $M(N)$ is the predicted moment force, $\rho\left(\mathrm{kg} / \mathrm{m}^{3}\right)$ is the fluid density, $A_{\mathrm{s}}\left(\mathrm{m}^{2}\right)$ is the rotor swept area.

In previous URANS simulation, (Castelli, et al., 2011) started the data sampling while the $C_{m}$ variations between two neighbouring revolutions is less than $1 \%$. Another study by (Rezaeiha, et al., 2018) found that after 20 revolutions the changes of $C_{m}$ and $C_{p}$ between two successive revolutions could be below $0.1 \%$ and $0.2 \%$ respectively, and between 20 and 100 revolutions, the differences over $C_{m}$ and $C_{p}$ would be lower than $1.06 \%$ and $2.41 \%$, respectively. While in agreement with those observations, the present study also found that after initial 20 revolutions, the $C_{m}$ variation reduced to less than $0.45 \%$ compared to previous revolution, indicating that a good convergence has been achieved.

However, due to the differences between the URANS and the SBES turbulence models, further test is needed to verify the revolution convergence of the SBES models. As shown in Figure 7, simulations have achieved convergence status after 34 revolutions for both OG and CG meshes. After this revolution, the difference of a verage power coefficient between two neighbouring revolutions is only $0.001 \%$. Hence, for all the remaining simulations, data retrieval will be collected from the $35^{\text {th }}$ revolution.

Compared to the URANS, SBES turbulence model will take more revolutions to reach convergence status. It is probably due to the fact that URANS turbulence models are mainly solving the mean flow and those large flow motions in the nearfield, and use ensemble a veraging solution in the far field (Salim, et al., 2013). In contrary, SBES turbulence model utilises the LES model in the far field, which can resolve the flow fluctuations to some extents and as a result, it will take longer time to achieve statistically converged flow field for both nearand far fields.

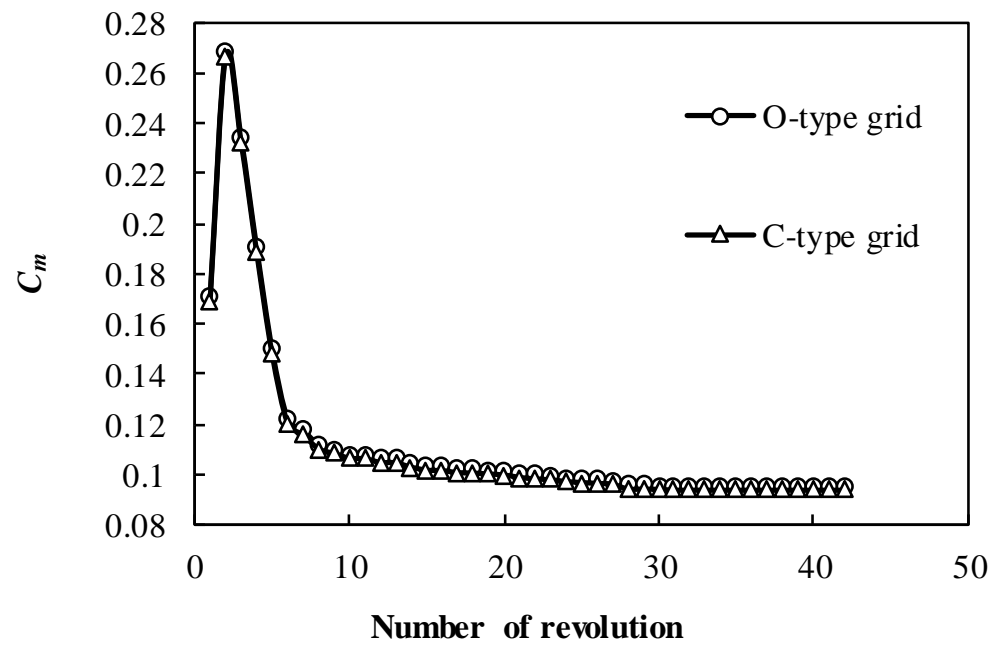

Figure 7. Moment coefficient changes over turbine revolution.

\subsection{Grid convergence}

The grid convergence study is conducted for simulation at $T S R=3.09$. At first, O-grid is considered with three grid resolutions from coarse, medium to finer meshes, each having 87, 174 and 348 cells around the blade. Then, C-grid is also tested using three grid resolutions with the same number of cells around the blade as the Ogrid.

Figures 8 and 9 illustrate the comparison of instantaneous moment coefficients over one revolution for both OG and CG meshes, respectively. As shown in the both figures, the moment coefficient changes along azimuthal positions have shown little difference between the medium and the fine grids while the coarse grid could not produce satisfying instantaneous moment coefficients. For both OG and CG, the average power coefficients of medium and fine grids are in good agreement with the experimental results of (Castelli, et al., 2011). Moreover, the relative error of a verage power coefficients between the medium and the fine grids is less than $4 \%$. Therefore, the medium grid has been chosen for the rest of simulations. 


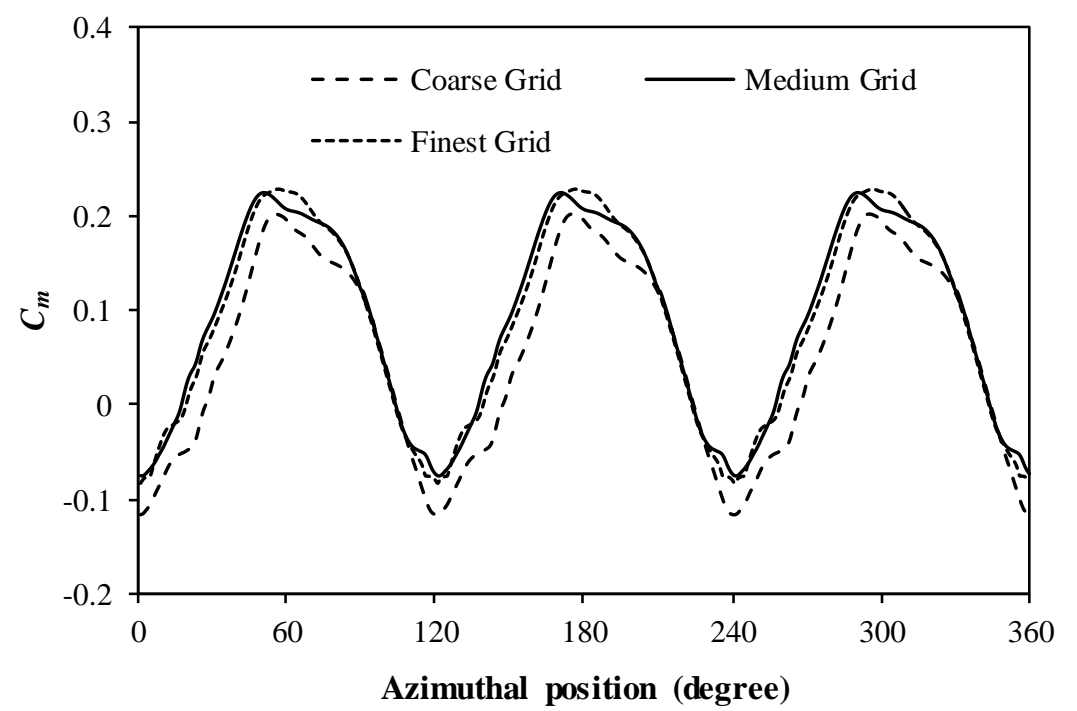

Figure 8. Comparison of instantaneous moment coefficients of VAWT with different grid resolutions for O-grid.

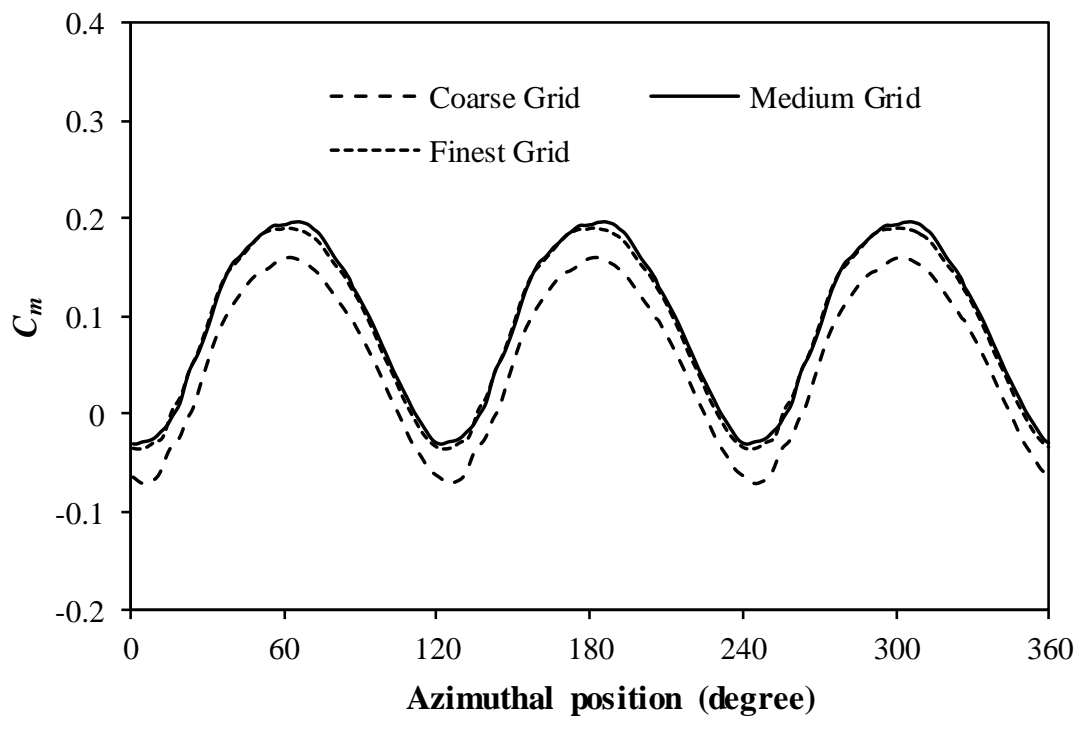

Figure 9. Comparison of instantaneous moment coefficients of VAWT with different grid resolutions for C-grid.

\subsection{Results validation}

For validating SBES results, simulations a re performed for TSR ranging from 1.44 to 3.3. Figure 10 shows the a verage $C_{p}$ prediction of current CFD predictions, a long with experimentaldata and CFD results of (Castelli, et al., 2011). Compared to CFD results of (Castelli, et al., 2011), the current CFD results using the same turbulence model of (Castelli, et al., 2011) (i.e. URANS realizable $k-\varepsilon$ with enhanced wall treatment) give better prediction especially in high TSR. However, these results still give large errors compared to experiment results.

On the other hand, it is found that present CFD prediction with OG and CG topologies using SBES turbulence model successfully reproduces the $C_{p}$ curve, especially the maximum peak value at an optimum TSR (2.64), is in very good agreement with the experiment of (Castelli, et al., 2011). It is clear that CFD using SBES turbulence model gives much better $C_{p}$ predictions than using URANS realizable $k-\varepsilon$ turbulence model with enhanced wall treatment.

Figures 11 and 12 illustrate the results comparison of the average power coefficient and the relative error between experiments and CFD results of (Castelli, et al., 2011) and present study over one revolution. As shown in Figure 11, even though the current CFD modelcan decrease the prediction error, the realizable $k-\varepsilon$ turbulence model with enhanced wall treatment still gives considerable error, especially at low TSR range. In fact, the angle 
between the blade zero lift line and the freestream direction (defined as absolute value of angle of attack, AoA) are relatively larger at low TSR than high TSR (Ma, et al., 2018). Moreover, the blades of VAWT can experience large range of $A O A$ at the same time. In addition, flow around blades could experience enormous large viscous region at low TSRs due to low Reynolds number effects (Lei, et al., 2017). Therefore, the blades will experience deep dynamic stall under such conditions. Because the $k-\varepsilon$ turbulence models family will over-predict the turbulence kinetic energy, it is unable to predict the deep dynamic stall at high AoA (Lei, 2005). As a result, URANS turbulence model produces large error at low TSRs.

Compared to the URANS, SBES turbulence model produces relatively smaller error in all range of TSRs for both O-grid and C-grid (see Figure 12). It is due to the fact that the large eddies in the farfield are resolved by the LES model in the SBES, while in URANS, the turbulence is treated as isotropic, leading to the momentum transport in the far field cannot be correctly considered. It is mentioned by (Warhaft, 2000) that this isotropic treatment can lead to reveala greater degree of intermittency (As example, URANS simplification make turbulent heat fluxes have basically no effect on the mean temperature. However, in fact, the turbulent fluctuations may generate significant difference properties in space and instantaneous properties in time (McDonough, 2007)). Furthermore, as discussed by (Lei, et al., 2017), DES turbulence model family can perform better due to its ability to produce realistic average wake velocity in the near and farfields.

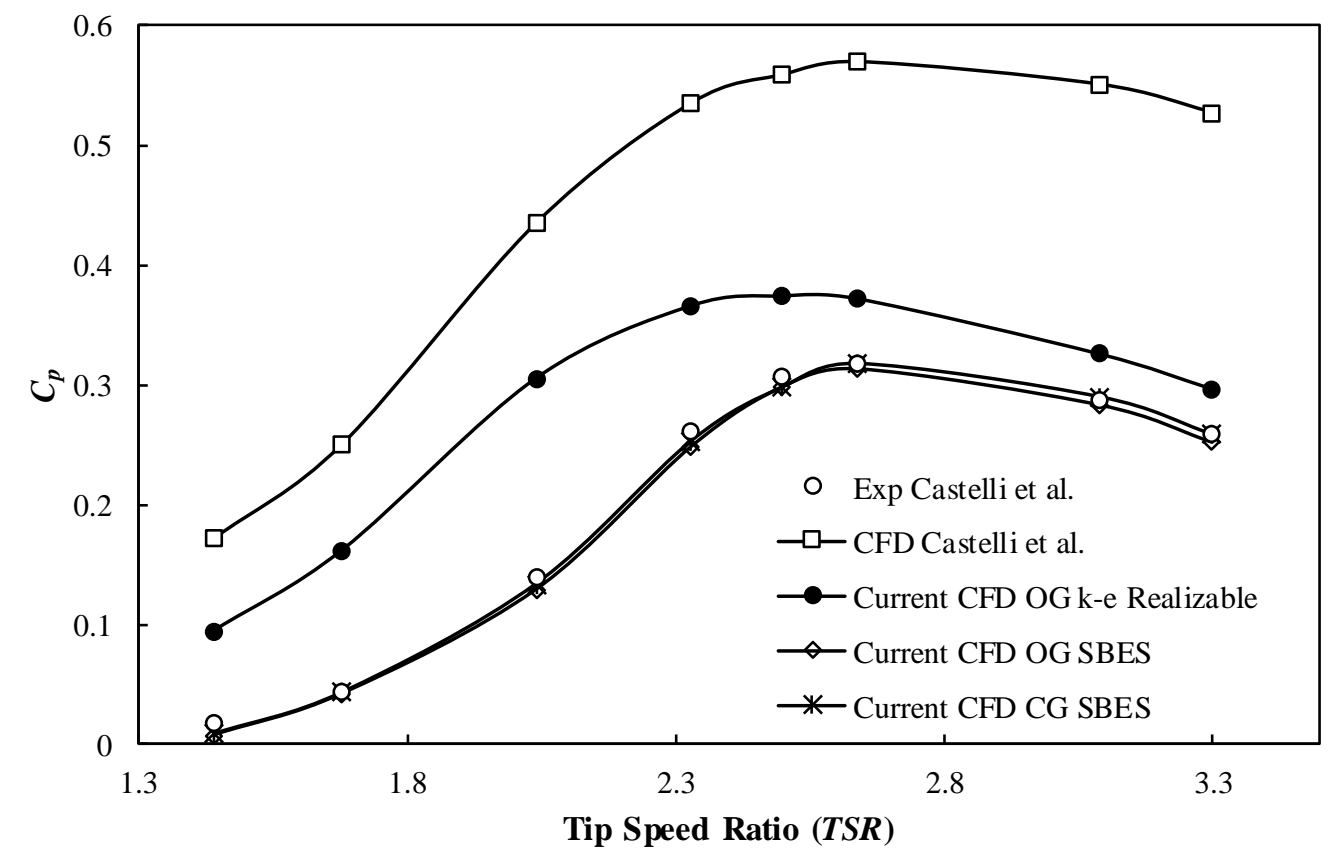

Figure 10. $C_{p}$ prediction comparison of current CFD and (Castelli, et al., 2011) models.

To understanding better ability of SBES turbulence models to predict $C_{p}$ of VAWT, the contour plots of $z$ vorticity at several important azimuthal positions of blade 1 is illustrated in Figure 11. It is shown that SBES turbulence model can predict stronger vortex shedding compared to $k-\varepsilon$ realizable turbulence model. It is also noticeable that compared to $k-\varepsilon$ realizable, SBES can show the development of dynamic stall and roll up trailing edge vortices. This ability lead to lower prediction of instantaneous $C_{m}$ value almost in all azimuthalposition and stronger fluctuation of this value after vortex shedding region therefore resulting lower prediction of $C_{p}$ (see Figure 12). 


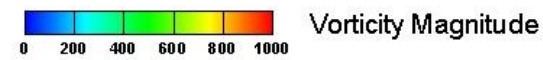

a) URANS (k- $\varepsilon$ realizable)

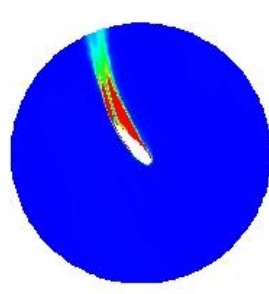

$\theta=135^{\circ}$

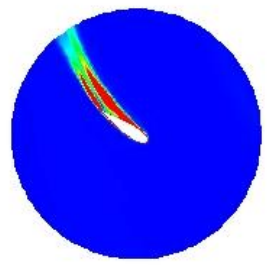

$\ominus=150^{\circ}$

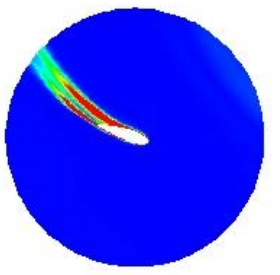

$\Theta=165^{\circ}$

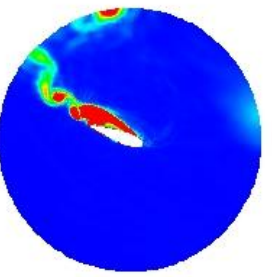

$\Theta=165^{\circ}$

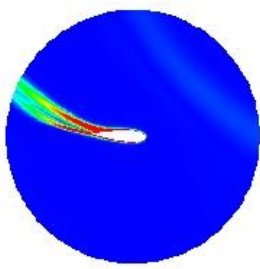

$\ominus=180^{\circ}$

b) Hybrid RANS-LES (SBES)

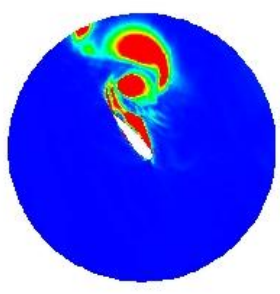

$\Theta=135^{\circ}$

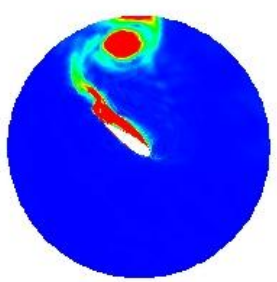

$\theta=150^{\circ}$

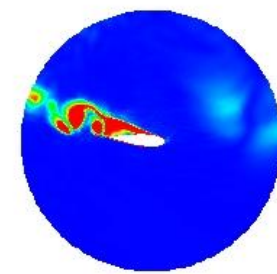

$\ominus=180^{\circ}$

Figure 11. Comparison of contour plots of $z$-vorticity, indicating the process of flow separation at important azimuthalpositions, prediction between SBES and $k-\varepsilon$ realizable turbulence models.

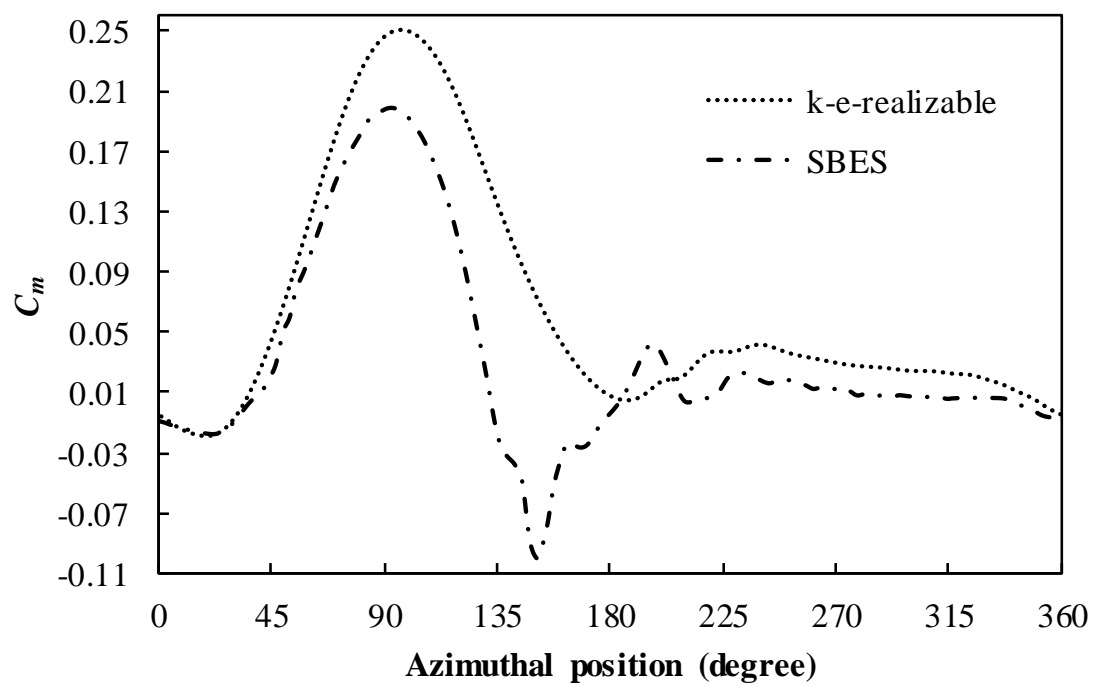

Figure 12. Comparison of instantaneous moment coefficient distribution of SBES and $k-\varepsilon$ realizable turbulence models. 
3.4 Grid topology study

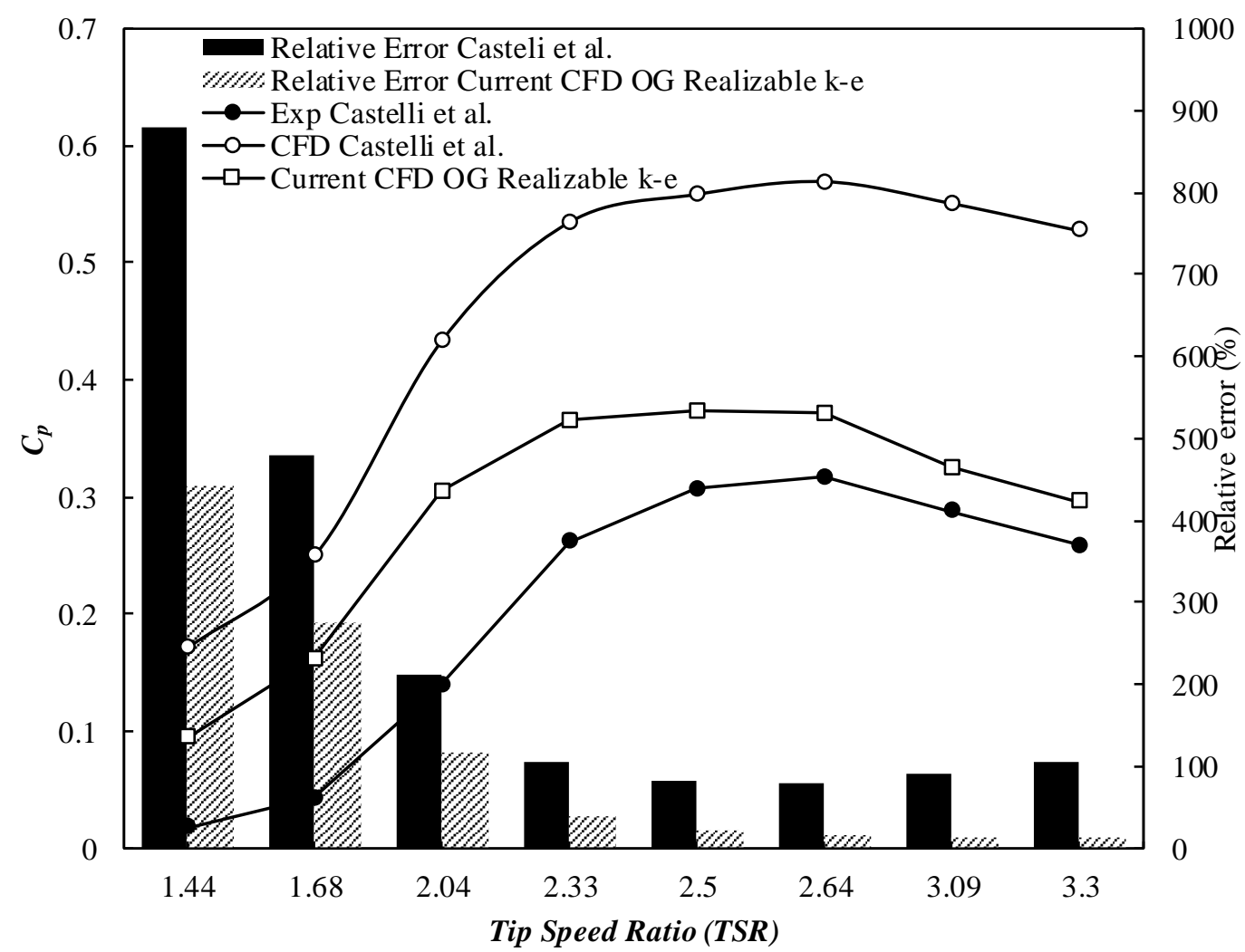

Figure 13. Comparison of a verage power coefficients between the experiment and simulation of (Castelli, et al., 2011) as well as relative errors in percentage.

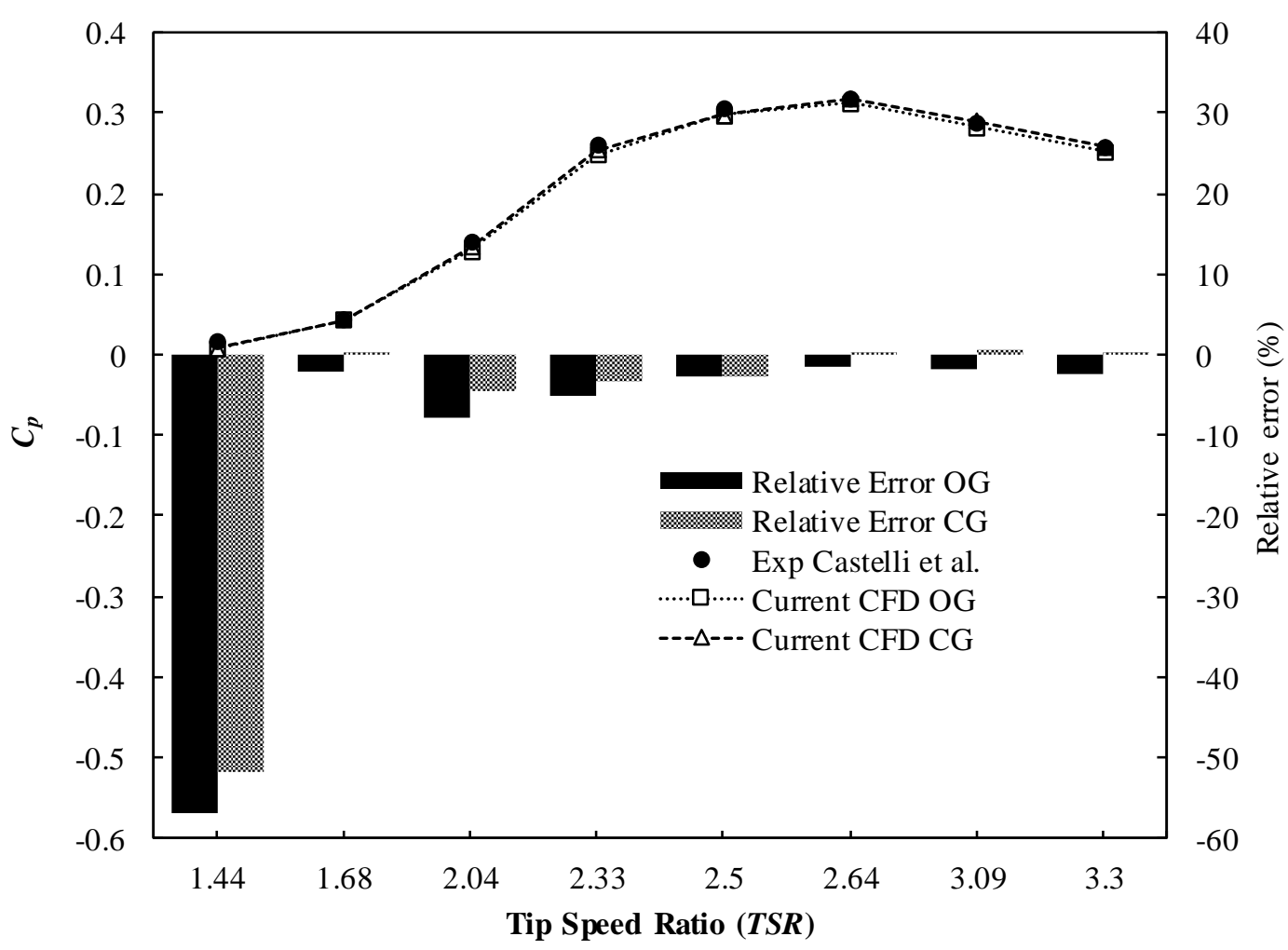

Figure 14. Comparison of a verage power coefficients between the experiment of (Castelli, et al., 2011) and current CFD simulation as well as relative errors in percentage. 
As depicted in Figures 13 and 14, there are clear differences in the time average $C_{p}$ distribution between CG and OG meshes, despite that the tendency of general behaviour of $C_{p}$ distribution is predicted consistently by the two grid topologies. Overall, the discrepancy between the OG and CG topologies is relatively minor if the time step is small enough as illustrated in Figures 13 and 14 respectively.

For all range of $T S R \mathrm{~s}$, simulations using the CG mesh produces relatively smaller error than that of OG, compared to experiment. This is probably contributed by the effect of grid density in the close region of the blade (i.e. control sub-domain) rather than the grid topology. Note that the CG control sub-domain contains more cells than OG (see Table 3) even though they have sa me number of cells a round the blade. Furthermore, DES turbulence modelfamily (including SBES) is relatively sensitive to grid resolutions not only in nearwall but also in farfield. As a result, simulation of CG mesh gives better prediction than OG. This observation is in agreement with a previous study of HAWT blade using DDES (Bangga, et al., 2017b).

Instantaneous moment coefficients of one representative blade (i.e. blade 1) over one revolution are plotted in Figure 15 for OG and CG meshes at $T S R=3.09$. Simulation of OG predicts earlier separation than CG, exhibited by earlier drop of $C_{m}$ value below zero at around $130^{\circ}$ azimuthalposition, while simulation of CG starts to have negative $C_{m}$ value later at about $150^{\circ}$ azimuthal position (see a dashed circle in Figure 15). This means that the starting point of no torque production (i.e. no power generation) predicted by simulation using OG mesh is earlier than that of CG mesh. However, the predicted recovery points (i.e. starting to produce positive torque again) are similar (around $190^{\circ}$ azimuthal position) and their behaviours after that point are almost identical. In addition, both simulations produce almost same maximum $C_{m}$ values at same azimuthal position. Due to these differences in prediction, the predicted power generation with OG mesh is slightly lower than CG and experimental measurements.

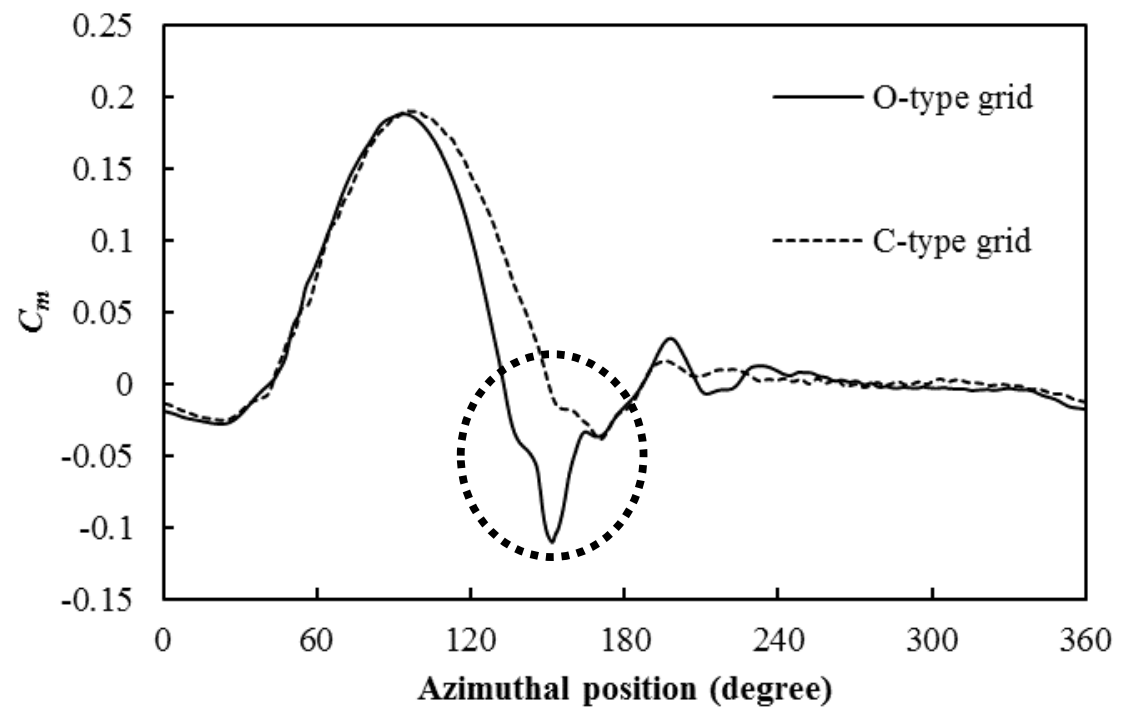

Figure 15. Comparison of instantaneous moment coefficient distribution of blade 1 for one turbine revolution between $\mathrm{O}$-grid and C-grid.

\section{Conclusions}

Two-dimensional CFD studies of Stress-blended Eddy Simulation (SBES) turbulence modelling to predict the performance of a three-straight-bladed VAWT and to evaluate grid topology effect on prediction accuracy have been carried out for Tip Speed Ratios (TSRs) ranging from 1.44 to 3.3 at a constant wind speed $9 \mathrm{~m} / \mathrm{s}$. The simulations are performed for 34 rotor revolutions to reach statistically converged results. After that, additional one revolution is simulated to collect data samples for a nalysis.

The results have shown that for both O-grid (OG) and C-grid (CG), SBES turbulence modelcan produce the power coefficients in a greement with experimentaldata (Castelli, et al., 2011). It is because SBES model resolves turbulence flow in the far field region with the time accurate Large Eddy Simulation (LES) model. Moreover, simulation with $\mathrm{CG}$ mesh produces better power coefficient $\left(C_{p}\right)$ distribution than OG due to higher grid density of $\mathrm{CG}$ in the near blade region. It is also found that an earlier flow separation has been predicted by simulation 
with $\mathrm{OG}$ mesh (at $130^{\circ}$ azimuthal position) than $\mathrm{CG}$ (at $150^{\circ}$ azimuthal position). However, both simulations using these two grids also predicted similar recovery points (i.e. around $190^{\circ}$ azimuthal position). Hence, it produces relatively lower $C_{p}$ than CG and experimentaldata (Castelli, et al., 2011). Further development of the present study will be carried out to perform 3D simulation in order to investigate the effect of 3D modelling contributing to the accuracy of CFD prediction using SBES model.

\section{Acknowledgement}

The first author would like to acknowledge the Ministry of Research, Technology and Higher Education of Indonesia and Indonesian Endowment Fund for Education (LPDP) of the Ministry of Finance of Indonesia for the financialsupport through BUDI LPDP scholarship.

\section{References}

Almohammadi, K. M., Ingham, D. B., Ma,L. \& Pourkashanian, M. (2015), "Modeling dynamic stall of a straight blade vertical axis wind turbine", Journal of Fluids and Structures, Vol. 57, pp. 144-158.

Arab, A., Javadi, M., Anbarsooz, M. \& Moghiman, M. (2017), "A numerical study on the aerodynamic performance and the self-starting characteristics of a Darrieus wind turbine considering its moment of inertia", Renewable Energy, Vol. 107,pp. 298-311.

Bangga, G., Hutomo, G., Wiranegara, R. \& Sasongko, H. (2017a), "Numerical study on a single bladed vertical axis wind turbine under dynamic stall", Journal of Mechanical Science and Technology, Vol. 31 No. 1, pp. 261267.

Bangga, G., Weihing, P., Lutz, T. \& Krämer, E. (2017b), "Effect of computational grid on accurate prediction of a wind turbine rotor using delayed detached-eddy simulations", Journal of Mechanical Science and Technology, Vol. 31 No. 5, pp. 2359-2364.

Cai, W., Li, Y. \& Liu, C. (2019), "Comparative Study of Scale-resolving Simulations for Marine-Propeller Unsteady Flows", International Communications in Heat and Mass Transfer, Vol. 100, pp. 1-11.

Castelli, M. R., Ardizzon, G., Battisti, L., Benini, E. \& Pavesi, G. (2010), "Modeling Strategy and Numerical Validation for a Darrieus Vertical Axis Micro-Wind Turbine", in Fluid Flow, Heat Transfer and Thermal Systems, Parts A and B Proceeding of ASME 2010 International Mechanical Engineering Congress and Exposition in Vancouver, British Columbia, Canada,2010, ASME, Vancouver, Vol. 7, pp. 409-418.

Castelli, M. R., Englaro, A. \& Benini, E. (2011), "The Darrieus wind turbine: Proposal for a new performance prediction model based on CFD", Energy, Vol. 36 No. 8, pp. 4919-4934.

Dabiri, J. O. (2011), "Potential order-of-magnitude enhancement of wind farm power density via counter-rotating vertical-axis wind turbine arrays", Journal of Renewable and Sustainable Energy, Vol. 3 No. 4, paper I.D. 043104.

Elkhoury, M., Kiwata, T. \& Aoun, E. (2015), "Experimental and numerical investigation of a three-dimensional vertical-axis wind turbine with variable-pitch", Journal of Wind Engineering \& Industrial Aerodynamics, Vol. 139, pp. 111-123.

Ferreira, C. J. S., van Zuijlen, A., Bijl, H., van Bussel, G. \& van Kuik, G. (2010), "Simulating dynamic stall in a two-dimensional vertical-axis wind turbine: verification and validation with particle image velocimetry data", Wind Energy, Vol. 13 No. 1, pp. 1-17.

Frank, T. \& Menter, F. (2017), "Validation of URANS SST and SBES in ANSYS CFD for the Turbulent Mixing of Two Parallel Planar Water Jets Impinging on a Stationary Pool", paper presented at ASME 2017 Verification and Validation Symposium, May 3-5, 2017, Las Vegas, Nevada, USA, available at: https://www.researchgate.net/publication/322570031_Validation_of_URANS_SST_and_SBES_in_ANSYS_CF D_for_the_Turbulent_Mixing_of_Two_Parallel_Planar_Water_Jets_Impinging_on_a_Stationary_PoolValidatio n_of_URANS_SST_and_SBES_in_ANSYS_CFD_for_the_Turbulent_M (accessed 10 January 2019).

Ghasemian, M., Ashrafi, Z. N. \& Sedaghat, A. (2017), "A review on computational fluid dynamic simulation techniques for Darrieus vertical axis wind turbines", Energy Conversion and Management, Vol. 149, pp. 87-100.

Ghasemian, M. \& Nejat, A. (2015), "Aero-acoustics prediction of a vertical axis wind turbine using Large Eddy Simulation and acoustic analogy", Energy, Vol. 88, pp. 711-717.

Lam, H. F. \& Peng, H. Y. (2016), "Study of wake characteristics of a vertical axis wind turbine by two - and threedimensional computationalfluid dynamics simulations", Renewable Energy, Vol. 90, pp. 386-398. 
Lanzafame, R., Mauro, S. \& Messina, M. (2014), "2D CFD Modeling of H-Darrieus Wind Turbines Using a Transition Turbulence Model", Energy Procedia, Vol. 45, pp. 131-140.

Lei, H., Zhou, D., Bao, Y., Li, Y. \& Han, Z. (2017), "Three-dimensional Improved Delayed Detached Eddy Simulation of a two-bladed vertical ax is wind turbine", Energy Conversion and Management, Vol. 133, pp. 235248.

Lei, Z. (2005), "Effect of RANS Turbulence Models on Computation of Vortical Flow over Wing-Body Configuration", Transactions of the Japan Society for Aeronautical and Space Sciences, Vol. 48, pp. 152-160.

Li, C., Zhu, S., Xu, Y. \& Xiao, Y. (2013), "2.5D large eddy simulation of vertical axis wind turbine in consideration of high angle of attack flow", Renewable Energy, Vol. 51, pp. 317-330.

Liu, C., Bu, W., Xu, D., Lei, Y. \& Li, X. (2017), "Application of Hybrid RANS/LES turbulence models in rotorstator fluid machinery: A comparative study", International Journal of Numerical Methods for Heat \& Fluid Flow, Vol. 27 No. 12, pp. 2717-2743.

Ma, N., Lei, H., Han, Z., Zhou, D., Bao, Y., Zhang, K., Zhou, L. \& Chen, C. (2018), "Airfoil optimization to improve power performance of a high-solidity vertical axis wind turbine at a moderate tip speed ratio", Energy, Vol. 150,pp. 236-252.

McDonough, J. M. (2007), "Introductory Lectures on Turbulences Physics, Mathematics and Modelling", Departments of MechanicalEngineering and Mathematics, University of Kentucky, Kentucky, USA.

Menter, F. (2018), "Stress-blended eddy simulation (SBES)—A new paradigm in hybrid RANS-LES modeling", Notes on Numerical Fluid Mechanics and Multidisciplinary Design, Vol. 137, pp. 27-37.

Mohamed, M. H., Ali, A. M. \& Hafiz, A. A. (2015), "CFD analysis for H-rotor Darrieus turbine as a low speed wind energy converter", Engineering Science and Technology, an International Journal, Vol. 18 No. 1, pp. 1-13.

Peng, H. Y. \& Lam,H. F. (2016), "Turbulence effects on the wake characteristics and aerodynamic performance of a straight-bladed vertical axis wind turbine by wind tunnel tests and large eddy simulations", Energy, Vol. 109, pp. 557-568.

Posa,A. \& Balaras, E. (2018), "Large Eddy Simulation of an isolated verticalaxis wind turbine", Journal of Wind Engineering \& Industrial Aerodynamics, Vol. 172, pp. 139-151.

Ravelli, S. \& Barigozzi, G. (2018a), "Dynamics of Coherent Structures and Random Turbulence in Pressure Side Film Cooling on a First Stage Turbine Vane", Journal of Turbomachinery, Vol. 141 No. 1, paper I.D. 011003.

Ravelli, S. \& Barigozzi, G. (2018b), "Stress-Blended Eddy Simulation of Coherent Unsteadiness in Pressure Side Film Cooling Applied to a First Stage Turbine Vane", Journal of Heat Transfer, Vol. 140 No. 9, paper I.D. 092201.

Rezaeiha, A., Montazeri, H. \& Blocken, B. (2018), "Towards accurate CFD simulations of vertical axis wind turbines at different tip speed ratios and solidities: Guidelines for azimuthal increment, domain size and convergence", Energy Conversion and Management, Vol. 156,pp. 301-316.

Salim, S. M., Ong, K. C. \& Cheah, S. C. (2013), "Comparison of RANS, URANS and LES in the Prediction of Airflow and Pollutant Dispersion", Lecture Notes in Electrical Engineering, Vol. 170, pp. 263-274.

Sutherland, H. J., Berg, D. E. \& Ashwill, T. D. (2012), "A retrospective of VAWT technology", Sandia National Laboratories, lbuquerque, New Mexico, USA.

Travin, A., Shur, M., Strelets, M. \& Spalart,P. (2000), "Detached-Eddy Simulations Past a Circular Cylinder", Flow, Turbulence and Combustion, Vol. 63, pp. 293-313.

Trivellato, F. \& Castelli, M. R. (2014), "On the Courant-Friedrichs-Lewy criterion of rotating grids in 2D verticalaxis wind turbine analysis", Renewable Energy, Vol. 62, pp. 53-62.

Wang, Y., Shen, S., G. Li, D. H. \& Zheng, Z. (2018), "Investigation on a erodynamic performance of verticalaxis wind turbine with different series airfoil shapes", Renewable Energy, Vol. 126, pp. 801-818.

Warhaft,Z. (2000). "Passive Scalars in Turbulent Flows", Annual Review of Fluid Mechanics, Vol. 32, pp. 203240 .

Zhu, H., Hao, W., Li, C. \& Ding, Q. (2018), "Simulation on flow control strategy of synthetic jet in a vertical axis wind turbine", Aerospace Science and Technology, Vol. 77, pp. 439-448. 